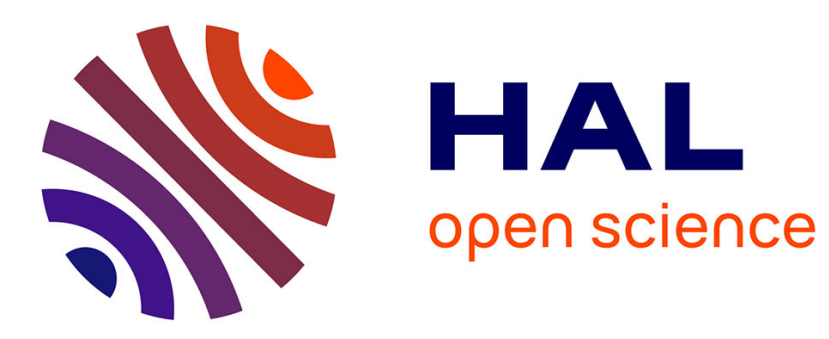

First Order Approach to

$L^{p}$

\title{
L p Estimates for the Stokes Operator on Lipschitz Domains
}

Alan Mcintosh, Sylvie Monniaux

\section{To cite this version:}

Alan Mcintosh, Sylvie Monniaux. First Order Approach to

$$
L^{p}
$$

L p Estimates for the Stokes Operator on Lipschitz Domains. 10th International International Society for Analysis, its Applications and Computation Congress (ISAAC 2015), Aug 2015, Macao, China. pp.55 - 75, 10.1007/978-3-319-41945-9_3 . hal-01474843

\section{HAL Id: hal-01474843 \\ https://hal.science/hal-01474843}

Submitted on 23 Feb 2017

HAL is a multi-disciplinary open access archive for the deposit and dissemination of scientific research documents, whether they are published or not. The documents may come from teaching and research institutions in France or abroad, or from public or private research centers.
L'archive ouverte pluridisciplinaire HAL, est destinée au dépôt et à la diffusion de documents scientifiques de niveau recherche, publiés ou non, émanant des établissements d'enseignement et de recherche français ou étrangers, des laboratoires publics ou privés. 


\title{
First Order Approach to $L^{p}$ Estimates for the Stokes Operator on Lipschitz domains
}

\author{
Alan McIntosh ${ }^{*} \quad$ Sylvie Monniaux ${ }^{\dagger}$
}

19th February 2016

\section{Introduction}

At the ISAAC meeting in Macau, the first author discussed the harmonic analysis of first order systems on bounded domains, with particular reference to his current joint research with the second author concerning the $L^{p}$ theory of Hodge-Dirac operators on Lipschitz domains, with implications for the Stokes' operator on such domains with Hodge boundary conditions. In this article, we present an overview of this material, staying with the three dimensional situation. Full definitions and proofs in higher dimensions can be found in [14]. In other papers with Marius Mitrea, the second author has pursued applications to the Navier-Stokes equation on Lipschitz domains. We will not comment further on that here, except to mention that the non-linear applications depend on having results for the linear Stokes operator in the case $p=3$ or possibly $p=3 / 2$ (the dual exponent to 3 ).

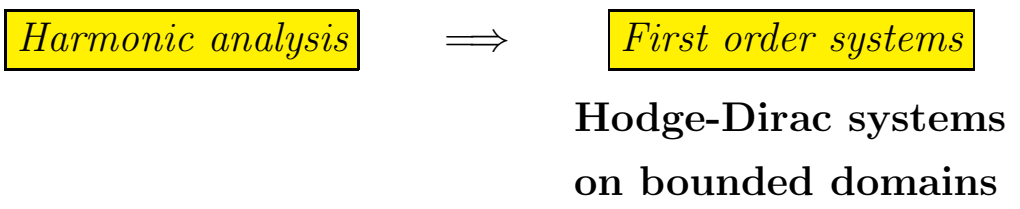

$\Downarrow$

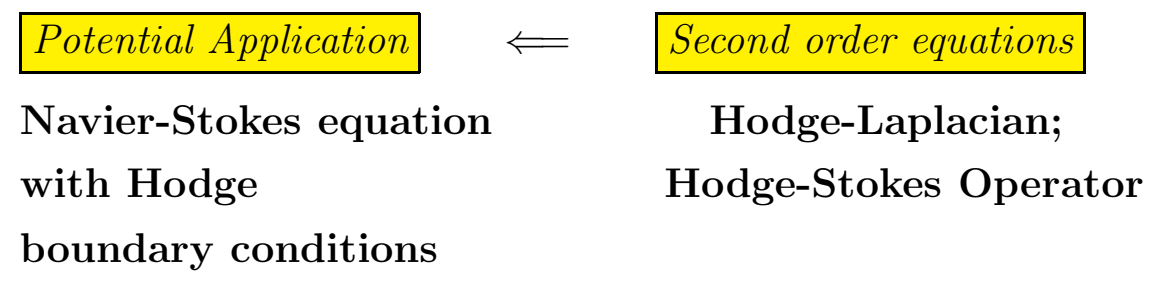

*Mathematical Sciences Institute, Australian National University, Canberra, ACT 2601, Australia - email: alan.mcintosh@anu.edu.au

†Aix-Marseille Université, CNRS, Centrale Marseille, I2M, UMR 7373, 13453 Marseille, France email: sylvie.monniaux@univ-amu.fr 


\section{Acknowledgments}

The first author would like to thank the organisers of the ISAAC meeting in Macau for arranging such an interesting conference, and in particular Tao Qian for his kind hospitality. The authors appreciate the support of the Mathematical Sciences Institute at the Australian National University, Canberra, where much of the collaboration took place, as well as the Laboratoire International Associé "Analysis and Geometry" and the Mathematical Institute in Marseille (I2M). Both authors were supported by the Australian Research Council.

\section{Hodge-Dirac operators}

Our aim is to investigate the $L^{p}$ theory of the first order Hodge-Dirac operator

$$
D_{H}=d_{\Omega}+\delta_{\bar{\Omega}}
$$

acting on a bounded domain $\Omega \subset \mathbb{R}^{3}$ satisfying some kind of Lipschitz condition.

Here $d_{\Omega}$ is the exterior derivative acting on differential forms in $L^{p}(\Omega, \Lambda)$, and $\delta_{\bar{\Omega}}$ is the adjoint operator which includes the tangential boundary condition

$$
\nu\lrcorner\left. u\right|_{\partial \Omega}=0
$$

i.e. the normal component of $u$ at the boundary $\partial \Omega$ is zero, at least on that part of the boundary where it is well-defined. This is effectively half a boundary condition for $D_{H}$, which is what is expected for a first order system.

Let us now define our terms.

\section{Lipschitz domains}

Henceforth $\Omega$ denotes a bounded connected open subset of $\mathbb{R}^{3}$, and $B$ denotes the unit ball in $\mathbb{R}^{3}$. We say that

- $\Omega$ is very weakly Lipschitz if $\Omega=\cup_{j=1}^{N}\left(\rho_{j} B\right)$ for some natural number $N$, where each map $\rho_{j}: B \rightarrow \rho_{j} B \subset \mathbb{R}^{3}$ is uniformly locally bilipschitz, and

$1=\sum_{j=1}^{N} \chi_{j}$ on $\Omega$, where each $\chi_{j}: \Omega \rightarrow[0,1]$ is a Lipschitz function with $\operatorname{sppt}_{\Omega}\left(\chi_{j}\right) \subset \rho_{j} B$

- $\Omega$ is strongly Lipschitz if, locally, the boundary $\partial \Omega$ of $\Omega$ is a portion of the graph of a Lipschitz function $g: \mathbb{R}^{2} \rightarrow \mathbb{R}$ (with respect to some rotated coordinate system), with $\Omega$ being to one side of the graph;

- $\Omega$ is smooth if each such function $g$ is smooth. 
In the above, $\operatorname{sppt}_{\Omega}\left(\chi_{j}\right)$ denotes the closure of $\left\{x \in \Omega ; \chi_{j}(x) \neq 0\right\}$ in $\Omega$.

Every strongly Lipschitz domain is weakly Lipschitz (which we shall not discuss further, but refer the reader to [5]) and every weakly Lipschitz domain is very weakly Lipschitz. A weakly Lipschitz domain which is not strongly Lipschitz is the well known two brick domain (consisting of one brick on top of another, pointing in orthogonal directions), and a very weakly Lipschitz domain which is not weakly Lipschitz is the unit ball with the half-disk $\left\{\left(x_{1}, x_{2}, x_{3}\right) \in B ; x_{3}=0, x_{1}>0\right\}$ removed.

In a strongly Lipschitz domain (and indeed in a weakly Lipschitz domain), there is a well-defined outward-pointing unit normal $\nu(y)$ for almost every $y \in \partial \Omega$. In fact $\nu \in L^{\infty}\left(\partial \Omega ; \mathbb{R}^{3}\right)$. As can be seen from the above example, the unit normal is not necessarily defined on the whole boundary of a very weakly Lipschitz domain.

\section{$5 \quad$ Exterior Algebra}

- The exterior algebra on $\mathbb{R}^{3}$ with basis $e_{1}, e_{2}, e_{3}$ is

$$
\begin{array}{rlrl}
\Lambda & =\Lambda^{0} \oplus \Lambda^{1} \oplus \Lambda^{2} \oplus \Lambda^{3} \approx \mathbb{C} \oplus \mathbb{C}^{3} \oplus \mathbb{C}^{3} \oplus \mathbb{C} \\
u & =u^{0}+u^{1}+u^{2}+u^{3} \quad \text { where } & \\
\Lambda^{0} & =\mathbb{C} & & \\
\Lambda^{1} & =\mathbb{C}^{3}: & u^{1}=u_{1}^{1} e_{1}+u_{2}^{1} e_{2}+u_{3}^{1} e_{3} & \\
\Lambda^{2} & \approx \mathbb{C}^{3}: & u^{2}=u_{2,3}^{2} e_{2} \wedge e_{3}+u_{3,1}^{2} e_{3} \wedge e_{1}+u_{1,2}^{2} e_{1} \wedge e_{2} \\
\Lambda^{3} & \approx \mathbb{C}: & u^{3}=u_{1,2,3}^{3} e_{1} \wedge e_{2} \wedge e_{3} \quad\left(e_{k} \wedge e_{j}=-e_{j} \wedge e_{k}\right)
\end{array}
$$

- $L^{p}(\Omega, \Lambda)=L^{p}(\Omega, \mathbb{C}) \oplus L^{p}\left(\Omega, \mathbb{C}^{3}\right) \oplus L^{p}\left(\Omega, \mathbb{C}^{3}\right) \oplus L^{p}(\Omega, \mathbb{C})$

- If $a=\sum_{j} a_{j} e_{j} \in \mathbb{R}^{3}, u \in \Lambda^{\ell}$, then $a \wedge u=\sum_{j} a_{j} e_{j} \wedge u \in \Lambda^{\ell+1}$

- If also $v \in \Lambda^{\ell+1}$ then $\left.a\right\lrcorner v \in \Lambda^{\ell}$ and $\left.\langle a \wedge u, v\rangle=\langle u, a\lrcorner v\right\rangle$

- $\left.\left.d u=\nabla \wedge u=\sum_{j} e_{j} \wedge \partial_{j} u, \quad \delta u=-\nabla\right\lrcorner u=-\sum_{j} e_{j}\right\lrcorner \partial_{j} u$

- The exterior product $\wedge$ and the contraction $\lrcorner$ can be represented by scalar multiplication, dot products and cross products.

\section{$6 \quad$ The de Rham complex on $\Omega \subset \mathbb{R}^{3}$}

Suppose that $\Omega$ denotes a bounded open subset of $\mathbb{R}^{3}$ and $1<p<\infty$.

The exterior derivative $d_{\Omega}$ defined on $\Omega$ can be expressed as follows:

$$
d_{\Omega}: 0 \rightarrow L^{p}(\Omega, \mathbb{C}) \stackrel{\nabla_{\Omega}}{\longrightarrow} L^{p}\left(\Omega, \mathbb{C}^{3}\right) \stackrel{\text { curl }_{\Omega}}{\longrightarrow} L^{p}\left(\Omega, \mathbb{C}^{3}\right) \stackrel{\operatorname{div}_{\Omega}}{\longrightarrow} L^{p}(\Omega, \mathbb{C}) \rightarrow 0
$$

(noting that curl is sometimes written as rot or $\nabla \times$, and div as $\nabla$.). 
As an operator, $d_{\Omega}: \mathcal{D}^{p}\left(d_{\Omega}\right) \rightarrow L^{p}(\Omega, \Lambda)$ is an unbounded operator with domain $\mathcal{D}^{p}\left(d_{\Omega}\right)=\left\{u \in L^{p}(\Omega, \Lambda) ; d_{\Omega} u \in L^{p}(\Omega, \Lambda)\right\}$.

Note that $d_{\Omega}{ }^{2}=0$ because $\operatorname{curl}_{\Omega} \nabla_{\Omega}=0$ and $\operatorname{div}_{\Omega} \operatorname{curl}_{\Omega}=0$, or as we can see directly, $d_{\Omega}{ }^{2} u=\sum_{j, k} e_{j} \wedge e_{k} \partial_{j} \partial_{k} u=0$ by the skew-symmetry of the wedge product.

Hence the range of $d_{\Omega}$ is contained in the null-space of $d_{\Omega}$, i.e. $\mathcal{R}^{p}\left(d_{\Omega}\right) \subset \mathcal{N}^{p}\left(d_{\Omega}\right)$ where $\mathcal{R}^{p}\left(d_{\Omega}\right)=\left\{v \in L^{p}(\Omega, \Lambda) ; v=d_{\Omega} u\right.$ for some $\left.u \in \mathcal{D}^{p}\left(d_{\Omega}\right)\right\}$ and $\mathcal{N}^{p}\left(d_{\Omega}\right)=\{u \in$ $\left.\mathcal{D}^{p}\left(d_{\Omega}\right) ; d_{\Omega} u=0\right\}$.

If $\Omega$ is very weakly Lipschitz, then $\overline{\mathcal{R}^{p}\left(d_{\Omega}\right)}=\mathcal{R}^{p}\left(d_{\Omega}\right)$ and the codimension of $\mathcal{R}^{p}\left(d_{\Omega}\right)$ in $\mathcal{N}^{p}\left(d_{\Omega}\right)$ is finite dimensional. We return to these facts in Section 20.

\section{The dual de Rham complex}

With $\Omega$ and $p$ as above, let $q=p^{\prime}$ (i.e. $\frac{1}{p}+\frac{1}{q}=1$ ).

The dual of the exterior derivative $d_{\Omega}: \mathcal{D}^{q}\left(d_{\Omega}\right) \rightarrow L^{q}(\Omega, \Lambda):$

$$
d_{\Omega}: 0 \rightarrow L^{q}(\Omega, \mathbb{C}) \stackrel{\nabla_{\Omega}}{\longrightarrow} L^{q}\left(\Omega, \mathbb{C}^{3}\right) \stackrel{\text { curl }_{\Omega}}{\longrightarrow} L^{q}\left(\Omega, \mathbb{C}^{3}\right) \stackrel{\operatorname{div}_{\Omega}}{\longrightarrow} L^{q}(\Omega, \mathbb{C}) \rightarrow 0
$$

is $\delta_{\bar{\Omega}}: \mathcal{D}^{p}\left(\delta_{\bar{\Omega}}\right) \rightarrow L^{p}(\Omega, \Lambda):$

$$
0 \leftarrow L^{p}(\Omega, \mathbb{C}) \stackrel{-\operatorname{div}_{\bar{\Omega}}}{\longleftarrow} L^{p}\left(\Omega, \mathbb{C}^{3}\right) \stackrel{\operatorname{curl}_{\bar{\Omega}}}{\longleftarrow} L^{p}\left(\Omega, \mathbb{C}^{3}\right) \stackrel{-\nabla_{\bar{\Omega}}}{\longleftarrow} L^{p}(\Omega, \mathbb{C}) \leftarrow 0: \delta_{\bar{\Omega}}
$$

where the domain $\mathcal{D}^{p}\left(\delta_{\bar{\Omega}}\right)$ is the completion of $C_{c}^{\infty}(\Omega, \Lambda)$ in the graph norm $\|u\|_{p}+$ $\left\|\delta_{\bar{\Omega}} u\right\|_{p}$.

Again, $\delta_{\bar{\Omega}}^{2}=0$, i.e. $\mathcal{R}^{p}\left(\delta_{\bar{\Omega}}\right) \subset \mathcal{N}^{p}\left(\delta_{\bar{\Omega}}\right)$.

If $\Omega$ is very weakly Lipschitz, then $\overline{\mathcal{R}^{p}\left(\delta_{\bar{\Omega}}\right)}=\mathcal{R}^{p}\left(\delta_{\bar{\Omega}}\right)$, with finite codimension in $\mathcal{N}^{p}\left(\delta_{\bar{\Omega}}\right)$.

If $\Omega$ is strongly Lipschitz, then the normal component of $u \in \mathcal{D}^{p}\left(\delta_{\bar{\Omega}}\right)$ at the boundary is zero, i.e.

$$
\left.\mathcal{D}^{p}\left(\delta_{\bar{\Omega}}\right)=\left.\left\{u \in L^{p}(\Omega, \Lambda) ; \delta_{\Omega} u \in L^{p}(\Omega, \Lambda), \nu\right\lrcorner u\right|_{\partial \Omega}=0\right\} .
$$

Remark 7.1. The condition $\nu\lrcorner\left. u\right|_{\partial \Omega}=0$ is to be understood in the following sense: for $u \in L^{p}(\Omega, \Lambda)$ such that $\delta_{\Omega} u \in L^{p}(\Omega, \Lambda)$ in a strongly Lipschitz domain, the normal component at the boundary $\nu\lrcorner\left. u\right|_{\partial \Omega}$ is defined as a functional on traces of differential forms $v \in W^{1, p^{\prime}}\left(\Omega, \Lambda\right.$ ) (where $\frac{1}{p^{\prime}}+\frac{1}{p}=1$ ) by the integration by parts formula:

$$
\langle\nu\lrcorner u, v\rangle_{\partial \Omega}=\langle u, d v\rangle_{\Omega}-\langle\delta u, v\rangle_{\Omega} .
$$

Since $\operatorname{Tr}_{\mid \partial \Omega}\left(W^{1, p^{\prime}}(\Omega, \Lambda)\right) \subseteq B_{1 / p}^{p^{\prime}, p^{\prime}}(\partial \Omega, \Lambda)$, we obtain that $\left.\nu\right\lrcorner u \in B_{-1 / p}^{p, p}(\partial \Omega, \Lambda)$. For more details, we refer to $[16$, §2.3].

Remark 7.2. Some care needs to be taken when consulting references, in that different authors use different sign conventions for $\delta$ and $\Delta$.

Remark 7.3. The definitions and results concerning very weakly Lipschitz domains in $\mathbb{R}^{3}$ can be adapted to domains in a Riemannian manifold with very little effort. 


\section{Hypothesis}

For the rest of this article, $\Omega$ denotes a very weakly Lipschitz domain in $\mathbb{R}^{3}$.

\section{The Hodge-Dirac operator $D_{H}=d_{\Omega}+\delta_{\bar{\Omega}}$ in $L^{2}(\Omega, \Lambda)$}

First we consider the case $p=2$. Then the exterior derivative $d_{\Omega}$ and adjoint interior derivative $\delta_{\bar{\Omega}}$ are unbounded operators in $L^{2}(\Omega, \Lambda)$ which satisfy

- $d_{\Omega}{ }^{2}=0, \quad \delta_{\bar{\Omega}}{ }^{2}=0, \quad d_{\Omega}{ }^{*}=\delta_{\bar{\Omega}}, \quad \delta_{\bar{\Omega}}{ }^{*}=d_{\Omega}$.

In $L^{2}(\Omega, \Lambda)$, define the Hodge-Dirac operator with tangential boundary condition $D_{H}:=d_{\Omega}+\delta_{\bar{\Omega}}$ with $\mathcal{D}^{2}\left(D_{H}\right)=\mathcal{D}^{2}\left(d_{\Omega}\right) \cap \mathcal{D}^{2}\left(\delta_{\bar{\Omega}}\right)$. It is straightforward to check the following properties (using the properties of $d_{\Omega}$ and $\delta_{\bar{\Omega}}$ just described):

- The Hodge-Dirac operator $D_{H}=d_{\Omega}+\delta_{\bar{\Omega}}$ is self-adjoint in $L^{2}(\Omega, \Lambda)$;

- $\mathcal{N}^{2}\left(D_{H}\right)=\mathcal{N}^{2}\left(d_{\Omega}\right) \cap \mathcal{N}^{2}\left(\delta_{\bar{\Omega}}\right)$ is finite-dimensional;

- The Hodge decomposition of $L^{2}(\Omega, \Lambda)$ takes the form

$$
\begin{aligned}
L^{2}(\Omega, \Lambda) & =\underset{\mathcal{N}}{ } \mathcal{N}^{2}\left(d_{\Omega}\right) \stackrel{\perp}{\oplus} \mathcal{R}^{2}\left(\delta_{\bar{\Omega}}\right) \\
L^{2}(\Omega, \Lambda) & =\mathcal{R}^{2}\left(d_{\Omega}\right) \stackrel{\perp}{\oplus} \mathcal{N}^{2}\left(\delta_{\bar{\Omega}}\right) \quad \text { and so } \\
L^{2}(\Omega, \Lambda) & =\mathcal{R}^{2}\left(d_{\Omega}\right) \stackrel{\perp}{\oplus} \mathcal{R}^{2}\left(\delta_{\bar{\Omega}}\right) \stackrel{\perp}{\oplus} \mathcal{N}^{2}\left(D_{H}\right) .
\end{aligned}
$$

- In particular, on restricting to the space of square integrable vector fields, $L^{2}\left(\Omega, \Lambda^{1}\right)=L^{2}\left(\Omega, \mathbb{C}^{3}\right)$, we have

$$
\begin{aligned}
L^{2}\left(\Omega, \Lambda^{1}\right) & =\mathcal{N}^{2}\left(\operatorname{curl}_{\Omega}\right) \stackrel{\perp}{\oplus} \mathcal{R}^{2}\left(\operatorname{curl}_{\bar{\Omega}}\right) \\
\cup & \cap \\
L^{2}\left(\Omega, \Lambda^{1}\right) & =\mathcal{R}^{2}\left(\nabla_{\Omega}\right) \stackrel{\perp}{\oplus} \mathcal{N}^{2}\left(\operatorname{div}_{\bar{\Omega}}\right)=\mathcal{H}^{2} \\
L^{2}\left(\Omega, \Lambda^{1}\right) & =\mathcal{R}^{2}\left(\nabla_{\Omega}\right) \stackrel{\perp}{\oplus} \mathcal{R}^{2}\left(\operatorname{curl}_{\bar{\Omega}}\right) \stackrel{\perp}{\oplus} \mathcal{N}^{2}\left(D_{H}\right)
\end{aligned}
$$

where $\mathcal{H}^{2}:=\mathcal{N}^{2}\left(\operatorname{div}_{\bar{\Omega}}\right) \subset L^{2}\left(\Omega, \Lambda^{1}\right)$.

In the case when $\Omega$ is strongly Lipschitz, $\mathcal{H}^{2}$ is the space of divergence-free square integrable vector fields which satisify the tangential boundary condition $\left.\nu \cdot u\right|_{\partial \Omega}=0$. 


\section{The Hodge-Laplacian $-\Delta_{H}=D_{H}{ }^{2}$}

In $L^{2}(\Omega, \Lambda)$, define the Hodge-Laplacian $-\Delta_{H}:=D_{H}^{2}=d_{\Omega} \delta_{\bar{\Omega}}+\delta_{\bar{\Omega}} d_{\Omega}$ with $\mathcal{D}^{2}\left(\Delta_{H}\right)=$ $\mathcal{D}^{2}\left(d_{\Omega} \delta_{\bar{\Omega}}\right) \cap \mathcal{D}^{2}\left(\delta_{\bar{\Omega}} d_{\Omega}\right)$. This is called the Hodge-Laplacian with absolute or generalised boundary conditions. We remark that $\Delta_{H}$ has the sign convention $\Delta_{H} u=\partial_{1}{ }^{2} u+$ $\partial_{2}{ }^{2} u+\partial_{3}{ }^{2} u, u \in \mathcal{D}^{2}\left(\Delta_{H}\right)$.

It is straightforward to check the following properties:

- The Hodge-Laplacian $-\Delta_{H}=d_{\Omega} \delta_{\bar{\Omega}}+\delta_{\bar{\Omega}} d_{\Omega}$ is non-negative self-adjoint in $L^{2}(\Omega, \Lambda)$;

- $\mathcal{N}^{2}\left(\Delta_{H}\right)=\mathcal{N}^{2}\left(D_{H}\right)=\mathcal{N}^{2}\left(d_{\Omega}\right) \cap \mathcal{N}^{2}\left(\delta_{\bar{\Omega}}\right)$

- The Hodge-Laplacian preserves each of the spaces $L^{2}\left(\Omega, \Lambda^{k}\right), 0 \leq k \leq 3$, and so splits as a direct sum of its restrictions to these spaces, as can be seen from the expression $-\Delta_{H}=d_{\Omega} \delta_{\bar{\Omega}}+\delta_{\bar{\Omega}} d_{\Omega}$ with:

$$
\begin{aligned}
& d_{\Omega}: 0 \underset{\leftarrow}{\rightarrow} L^{2}(\Omega, \mathbb{C}) \stackrel{\nabla_{\Omega}}{\rightarrow} \underset{\leftarrow}{\leftarrow} L^{2}\left(\Omega, \mathbb{C}^{3}\right) \stackrel{\operatorname{curl}_{\Omega}}{\rightarrow} L^{2}\left(\Omega, \mathbb{C}^{3}\right) \stackrel{\operatorname{div}_{\Omega}}{\leftarrow} L^{2}(\Omega, \mathbb{C}) \underset{\leftarrow}{\leftarrow} 0: \delta_{\bar{\Omega}} \\
& \begin{array}{cccc}
\leftarrow \operatorname{div}_{\bar{\Omega}} & \cup & \leftarrow & \leftarrow \\
\mathcal{H}^{2} & \operatorname{curl}_{\bar{\Omega}} & -\nabla_{\bar{\Omega}}
\end{array}
\end{aligned}
$$$$
\begin{array}{r}
-\Delta_{H}=-\operatorname{div}_{\bar{\Omega}} \nabla_{\Omega} \oplus\left(-\nabla_{\Omega} \operatorname{div}_{\bar{\Omega}}+\operatorname{curl}_{\bar{\Omega}} \operatorname{curl}_{\Omega}\right) \oplus\left(\operatorname{curl}_{\Omega} \operatorname{curl}_{\bar{\Omega}}-\nabla_{\bar{\Omega}} \operatorname{div}_{\Omega}\right) \oplus-\operatorname{div}_{\Omega} \nabla_{\bar{\Omega}} \\
\left(=-\Delta_{\text {Neumann }}\right) \\
\left(=-\Delta_{\text {Dirichlet }}\right)
\end{array}
$$

Indeed it also preserves each component of the Hodge decomposition, in particular $\mathcal{H}^{2}=L^{2}\left(\Omega, \Lambda^{1}\right) \cap \mathcal{N}^{2}\left(\delta_{\bar{\Omega}}\right)=\mathcal{N}^{2}\left(\operatorname{div}_{\bar{\Omega}}\right)$.

\section{The Hodge-Stokes operator $S_{H}=-\left.\Delta_{H}\right|_{\mathcal{H}^{2}}$}

In $\mathcal{H}^{2}$, define the Stokes operator with Hodge boundary conditions by $S_{H} u=-\Delta_{H} u=$ $\operatorname{curl}_{\bar{\Omega}} \operatorname{curl}_{\Omega} u, u \in \mathcal{H}^{2}$ (i.e. $\left.\operatorname{div}_{\bar{\Omega}} u=0\right)$ with $\mathcal{D}^{2}\left(S_{H}\right)=\left\{u \in L^{2}\left(\Omega, \Lambda^{1}\right) ; \operatorname{div}_{\bar{\Omega}} u=\right.$ $\left.0, \operatorname{curl}_{\bar{\Omega}} \operatorname{curl}_{\Omega} u \in L^{2}\left(\Omega, \Lambda^{1}\right)\right\}$. It is straightforward to check the following properties:

- The Hodge-Stokes operator $S_{H}=\operatorname{curl}_{\bar{\Omega}} \operatorname{curl}_{\Omega}$ is non-negative self-adjoint in $\mathcal{H}^{2}$;

- $\mathcal{N}^{2}\left(S_{H}\right)=\mathcal{N}^{2}\left(D_{H}\right) \cap L^{2}\left(\Omega, \Lambda^{1}\right)=\mathcal{N}^{2}\left(\operatorname{curl}_{\Omega}\right) \cap \mathcal{N}^{2}\left(\operatorname{div}_{\bar{\Omega}}\right)$ is finite-dimensional;

If $\Omega$ is strongly Lipschitz and $u \in \mathcal{D}^{2}\left(S_{H}\right)$, then the tangential boundary conditions $\nu .\left.u\right|_{\partial \Omega}=0 ; \nu \times\left.\operatorname{curl} u\right|_{\partial \Omega}=0$ hold. See, e.g., [17, §3]. 


\section{$12 L^{2}$ results for $D_{H}, \Delta_{H}$ and $S_{H}$}

To summarise, we have the following properties:

- $L^{2}(\Omega, \Lambda)=\mathcal{R}^{2}\left(d_{\Omega}\right) \stackrel{\perp}{\oplus} \mathcal{R}^{2}\left(\delta_{\bar{\Omega}}\right) \stackrel{\perp}{\oplus} \mathcal{N}^{2}\left(D_{H}\right)$;

- Hodge-Dirac operator $D_{H}=d_{\Omega}+\delta_{\bar{\Omega}}$ is self-adjoint in $L^{2}(\Omega, \Lambda)$;

- Hodge-Laplacian $-\Delta_{H}=D_{H}{ }^{2}=d_{\Omega} \delta_{\bar{\Omega}}+\delta_{\bar{\Omega}} d_{\Omega}$ is non-negative self-adjoint in $L^{2}(\Omega, \Lambda)$

- Hodge-Stokes operator $S_{H}=-\left.\Delta_{H}\right|_{\mathcal{H}^{2}}$ is non-negative self-adjoint in $\mathcal{H}^{2}=$ $\mathcal{N}^{2}\left(\operatorname{div}_{\bar{\Omega}}\right)$.

So $D_{H}, \Delta_{H}, S_{H}$ all have resolvent bounds, e.g.

$$
\begin{aligned}
\left\|\left(I+i t D_{H}\right)^{-1} u\right\|_{2} \leq\|u\|_{2} & \forall u \in L^{2}(\Omega, \Lambda), & \forall t \in \mathbb{R} \backslash\{0\} \\
\left\|\left(I-t^{2} \Delta_{H}\right)^{-1} u\right\|_{2} \leq\|u\|_{2} & \forall u \in L^{2}(\Omega, \Lambda), & \forall t>0 \\
\left\|\left(I+t^{2} S_{H}\right)^{-1} u\right\|_{2} \leq\|u\|_{2} & \forall u \in \mathcal{H}^{2} \quad, & \forall t>0
\end{aligned}
$$

and all have functional calculi of self-adjoint operators, in particular

$$
\left\|D_{H} u\right\|_{2}=\left\|\operatorname{sgn}\left(D_{H}\right) \sqrt{-\Delta_{H}} u\right\|_{2}=\left\|\sqrt{-\Delta_{H}} u\right\|_{2} \quad \forall u \in \mathcal{D}^{2}\left(D_{H}\right)=\mathcal{D}^{2}\left(\sqrt{-\Delta_{H}}\right) .
$$

\section{$13 L^{p}$ questions for $D_{H}, \Delta_{H}$ and $S_{H}, 1<p<\infty$}

Whether or not the $L^{p}$ versions of these properties hold, depends on $\Omega$ and $p$. Of course, we no longer have orthogonality of the Hodge decomposition, and the constants in the resolvent bounds and the functional calculi may depend on $p$. Allowing for this, when $\Omega$ is smooth, all of the properties hold for all $p \in(1, \infty)$.

In our situation, namely when $\Omega$ is a very weakly Lipschitz domain, we list the main properties and then discuss their relationship with one another, and conditions under which they hold.

$\left(H_{p}\right) \quad D_{H}$ has an $L^{p}$ Hodge decomposition: $L^{p}(\Omega, \Lambda)=\mathcal{R}^{p}\left(d_{\Omega}\right) \oplus \mathcal{R}^{p}\left(\delta_{\bar{\Omega}}\right) \oplus \mathcal{N}^{p}\left(D_{H}\right)$;

$\left(R_{p}\right) \quad D_{H}$ is bisectorial in $L^{p}$, in particular $\left\|\left(I+i t D_{H}\right)^{-1} u\right\|_{p} \leq C\|u\|_{p} \quad \forall t \in \mathbb{R} \backslash\{0\}$;

$\left(F_{p}\right) \quad D_{H}$ has a bounded $H^{\infty}\left(S_{\mu}^{o}\right)$ functional calculus in $L^{p}(\Omega, \Lambda)$ for all $\mu>0$ :

$\left\|f\left(D_{H}\right) u\right\|_{p} \leq C_{\mu}\|f\|_{\infty}\|u\|_{p} \forall f \in H^{\infty}\left(S_{\mu}^{o}\right)$, in particular, $\left\|D_{H} u\right\|_{p} \approx\left\|\sqrt{-\Delta_{H}} u\right\|_{p}$.

Here $S_{\mu}^{o}=\{z \in \mathbb{C} ;|\arg z|<\mu$ or $|\arg (-z)|<\mu\}, 0<\mu<\pi / 2$.

Let us note that:

- $\left(F_{p}\right) \Longrightarrow\left(H_{p}\right)$ : Exercise. 
- $\left(F_{p}\right) \Longrightarrow\left(R_{p}\right) \Longrightarrow$ Hodge-Laplacian is sectorial in $L^{p}(\Omega, \Lambda)$, in particular $\Delta_{H}$ has the $L^{p}$ resolvent bounds

$$
\left\|\left(I-t^{2} \Delta_{H}\right)^{-1} u\right\|_{p}=\left\|\left(I+i t D_{H}\right)^{-1}\left(I-i t D_{H}\right)^{-1} u\right\|_{p} \leq C^{2}\|u\|_{p} \quad \forall t>0 .
$$

- $\left(F_{p}\right) \Longrightarrow$ Hodge-Laplacian has a bounded $H^{\infty}\left(S_{\mu}^{o}\right)$ functional calculus $\forall \mu>0$ $\Longrightarrow$ maximal regularity results for the parabolic equation (see $\S 14$ )

$$
\begin{aligned}
\partial_{t} F(t, .)-\Delta_{H} F(t, .) & =h(t, .) \in L^{q}\left((0, T) ; L^{p}(\Omega, \Lambda)\right), t>0 \\
F(0, .) & =0 .
\end{aligned}
$$

\section{Background on bisectorial operators and holo- mophic functional calculus}

If the reader maintains attention on the resolvent bounds stated for the Hodge-Dirac operator, the Hodge-Laplacian and the Hodge-Stokes operator, then this material is not needed. But we will briefly describe the above-mentioned concepts for those who are interested.

Let $0 \leq \omega<\mu<\frac{\pi}{2}$. Define closed and open sectors and double sectors in the complex plane by

$$
\begin{aligned}
S_{\omega+} & :=\{z \in \mathbb{C}:|\arg z| \leq \omega\} \cup\{0\}, \quad S_{\omega-}:=-S_{\omega+}, \\
S_{\mu+}^{o} & :=\{z \in \mathbb{C}: z \neq 0,|\arg z|<\mu\}, \quad S_{\mu-}^{o}:=-S_{\mu+}^{o}, \\
S_{\omega} & :=S_{\omega+} \cup S_{\omega-}, \quad S_{\mu}^{o}:=S_{\mu+}^{o} \cup S_{\mu-}^{o} .
\end{aligned}
$$

Let $0 \leq \omega<\frac{\pi}{2}$. A closed operator $D$ acting on a closed subspace $\mathcal{X}^{p}$ of $L^{p}(\Omega, \Lambda)$ is called bisectorial with angle $\omega$ if its spectrum $\sigma(D) \subset S_{\omega}$, and for all $\theta \in\left(\omega, \frac{\pi}{2}\right)$ there exists $C_{\theta}>0$ such that

$$
\left\|\lambda(\lambda I-D)^{-1} u\right\|_{p} \leq C_{\theta}\|u\|_{p} \quad \forall \lambda \in \mathbb{C} \backslash S_{\theta}, \forall u \in \mathcal{X}^{p}
$$

In $\left(R_{p}\right)$, we really mean that $D_{H}$ is bisectorial with angle 0 , and present the particular resolvent bounds for $\lambda=i / t$ with $t$ real.

Let $0 \leq \omega<\pi$. A closed operator $D$ acting on $\mathcal{X}^{p}$ is called sectorial with angle $\omega$ if $\sigma(D) \subset S_{\omega+}$, and for all $\theta \in(\omega, \pi)$ there exists $C_{\theta}>0$ such that

$$
\left\|\lambda(\lambda I-D)^{-1} u\right\|_{p} \leq C_{\theta}\|u\|_{p} \quad \forall \lambda \in \mathbb{C} \backslash S_{\theta+}, \forall u \in \mathcal{X}^{p}
$$

For the Hodge-Laplacian, we really mean sectorial with angle 0 , and present the particular resolvent bounds for $\lambda=-1 / t^{2}$ with $t>0$.

Denote by $H^{\infty}\left(S_{\mu}^{o}\right)$ the space of all bounded holomorphic functions on $S_{\mu}^{o}$, and by $\Psi\left(S_{\mu}^{o}\right)$ the subspace of those functions $\psi$ which satisfy $|\psi(z)| \leq C \min \left\{|z|^{\alpha},|z|^{-\alpha}\right\}$ for some $\alpha>0$. Similarly define $H^{\infty}\left(S_{\mu+}^{o}\right)$ and $\Psi\left(S_{\mu+}^{o}\right)$. 
For $D$ bisectorial with angle $\omega$ in $\mathcal{X}^{p}$ and $\psi \in \Psi\left(S_{\mu}^{o}\right), \omega<\mu<\frac{\pi}{2}$ (or sectorial with angle $\omega$ and $\left.\psi \in \Psi\left(S_{\mu+}^{o}\right), \omega<\mu<\pi\right)$ define $\psi(D)$ through the Cauchy integral

$$
\psi(D) u=\frac{1}{2 \pi i} \int_{\gamma} \psi(z)(z I-D)^{-1} u d z, \quad u \in \mathcal{X}^{p},
$$

where $\gamma$ denotes the boundary of $S_{\theta}$ (or $S_{\theta+}$ ) for some $\theta \in(\omega, \mu)$, oriented counterclockwise. Then $D$ is said to have a bounded holomorphic functional calculus with angle $\mu$, or a bounded $H^{\infty}\left(S_{\mu}^{o}\right)$ (or $\left.H^{\infty}\left(S_{\mu+}^{o}\right)\right)$ functional calculus in $\mathcal{X}^{p}$ if there exists $C>0$ such that

$$
\|\psi(D) u\|_{p} \leq C_{p}\|\psi\|_{\infty}\|u\|_{p} \quad \forall u \in \mathcal{X}^{p}, \forall \psi \in \Psi\left(S_{\mu}^{o}\right)\left(\text { or } \Psi\left(S_{\mu+}^{o}\right)\right) .
$$

For such an operator, the functional calculus extends to all $f \in H^{\infty}\left(S_{\mu}^{o}\right)$ (or $\left.H^{\infty}\left(S_{\mu+}^{o}\right)\right)$ on defining

$$
f(D) u=\lim _{n \rightarrow \infty} \psi_{n}(D) u, \quad u \in \mathcal{X}^{p},
$$

where the functions $\psi_{n} \in \Psi\left(S_{\mu}^{o}\right)$ are uniformly bounded and tend locally uniformly to $f$. (We are implicitly taking $f(0)=0$ here.)

We list some properties.

- If $D$ is bisectorial of angle $\omega<\pi / 2$, then $D^{2}$ is sectorial of angle $2 \omega<\pi$.

- If $D$ has a bounded $H^{\infty}\left(S_{\mu}^{o}\right)$ functional calculus, then $D^{2}$ has a bounded $H^{\infty}\left(S_{2 \mu+}^{o}\right)$ functional calculus.

- If $D$ is a bisectorial operator with a bounded holomorphic functional calculus in $\mathcal{X}^{p}$, then $\|\operatorname{sgn}(D) u\|_{p} \leq C_{p}\|u\|_{p}$ for all $u \in \mathcal{X}^{p}$ where

$$
\operatorname{sgn}(z)= \begin{cases}-1 & z \in S_{\mu-}^{o} \\ 0 & z=0 \\ +1 & z \in S_{\mu+}^{o}\end{cases}
$$

and so $D$ has Riesz transform bounds in $\mathcal{X}^{p}$ :

$$
\begin{aligned}
\|D u\|_{p} & =\left\|\operatorname{sgn}(D) \sqrt{D^{2}} u\right\|_{p} \leq C_{p}\left\|\sqrt{D^{2}} u\right\|_{p} \\
\left\|\sqrt{D^{2}} u\right\|_{p} & =\|\operatorname{sgn}(D) D u\|_{p} \leq C_{p}\|D u\|_{p}, \quad u \in \mathcal{D}(D)=\mathcal{D}\left(\sqrt{D^{2}}\right) .
\end{aligned}
$$

- If $S$ is a sectorial operator with a bounded holomorphic functional calculus of angle $<\pi / 2$ in $\mathcal{X}^{p}$, and $1<q<\infty, 0<T \leq \infty$, then the parabolic equation

$$
\begin{aligned}
\partial_{t} F(t, .)+S F(t, .) & =h(t, .) \in L^{q}\left((0, T) ; \mathcal{X}^{p}\right), t>0 \\
F(0, .) & =0
\end{aligned}
$$

has maximal regularity in the sense that

$$
\left\{\int_{0}^{T}\|F(t, .)\|_{p}^{q} d t\right\}^{1 / q}+\left\{\int_{0}^{T}\|S F(t, .)\|_{p}^{q} d t\right\}^{1 / q} \leq C_{p, q}\left\{\int_{0}^{T}\|h(t, .)\|_{p}^{q} d t\right\}^{1 / q} .
$$


For further details on the above material, see [13, 8, 2] or the lecture notes [1, 12].

Solution to Exercise. Show that $\left(H_{p}\right)$ is a consequence of $\left\|D_{H} u\right\|_{p} \approx\left\|\sqrt{-\Delta_{H}} u\right\|_{p}$. We need $\left\|D_{H} u\right\|_{p} \approx\left\|d_{\Omega} u\right\|_{p}+\left\|\delta_{\bar{\Omega}} u\right\|_{p}$, or equivalently $\left\|d_{\Omega} u\right\|_{p} \lesssim\left\|D_{H} u\right\|_{p}$.

Write $u=\sum_{k=0}^{3} u^{k}, u^{k} \in L^{p}\left(\Omega, \Lambda^{k}\right)$, then

$$
\begin{aligned}
\left\|d_{\Omega} u\right\|_{p} & \approx \sum_{\ell=0}^{3}\left\|\left(d_{\Omega} u\right)^{\ell}\right\|_{p}=\sum_{k=0}^{3}\left\|d_{\Omega}\left(u^{k}\right)\right\|_{p} \leq \sum_{k=0}^{3}\left\|D_{H}\left(u^{k}\right)\right\|_{p} \approx \sum_{k=0}^{3}\left\|\sqrt{-\Delta_{H}}\left(u^{k}\right)\right\|_{p} \\
& =\sum_{k=0}^{3}\left\|\left(\sqrt{-\Delta_{H}} u\right)^{k}\right\|_{p} \approx\left\|\sqrt{-\Delta_{H}} u\right\|_{p} \approx\left\|D_{H} u\right\|_{p} .
\end{aligned}
$$

(The bound $\left\|d_{\Omega}\left(u^{k}\right)\right\|_{p} \leq\left\|d_{\Omega}\left(u^{k}\right)+\delta_{\bar{\Omega}}\left(u^{k}\right)\right\|_{p}$ holds because $d_{\Omega}\left(u^{k}\right) \in L^{p}\left(\Omega, \Lambda^{k+1}\right)$ and $\delta_{\bar{\Omega}}\left(u^{k}\right) \in L^{p}\left(\Omega, \Lambda^{k-1}\right)$.) The idea for this result comes from [3, $\left.\S 5\right]$.

\section{$15 \quad L^{p}$ Hodge decomposition}

It is a consequence of the interpolation properties of the spaces $\mathcal{R}^{p}\left(d_{\Omega}\right)$ and $\mathcal{R}^{p}\left(\delta_{\bar{\Omega}}\right)$ (see Remark 20.2) that property $\left(H_{p}\right)$ is stable in $p$ in the following sense.

Theorem 15.1. There exist Hodge exponents $p_{H}, p^{H}=p_{H}{ }^{\prime}$ with $1 \leq p_{H}<2<$ $p^{H} \leq \infty$ such that the Hodge decomposition $\left(H_{p}\right)$

$$
L^{p}(\Omega, \Lambda)=\mathcal{R}^{p}\left(d_{\Omega}\right) \oplus \mathcal{R}^{p}\left(\delta_{\bar{\Omega}}\right) \oplus \mathcal{N}^{p}\left(D_{H}\right)
$$

holds in the $L^{p}$ norm if and only if $p_{H}<p<p^{H}$.

This is proved in $[14, \S 4]$, following a similar proof in $[11, \S 3.2]$.

It is well known that, when $\Omega$ has smooth boundary, then $p_{H}=1$ and $p^{H}=\infty$. See, e.g., [18, Theorem 2.4.2 and 2.4.14] for the general case of smooth compact Riemannian manifolds with boundary.

If $\Omega$ is a strongly Lipschitz domain in $\mathbb{R}^{3}$, then $p_{H}<3 / 2<3<p^{H}$. See, e.g., [15, Theorem 1.1]. In [14] we reprove this result, with the new techniques having the advantage of providing a new result in higher dimensions, namely that $p_{H}<$ $2 n /(n+1)<2 n /(n-1)<p^{H}$ when $\Omega$ is a bounded strongly Lipschitz domain in $\mathbb{R}^{n}$. In fact we show that $D_{H}$ has a bounded holomorphic functional calculus in $L^{p}(\Omega, \Lambda)$ for some $p<2 n /(n+1)$ (and hence, by duality, in $L^{p^{\prime}}(\Omega, \Lambda)$ ), and apply the Exercise in $\S 13$.

\section{$16 L^{p}$ results for $D_{H}, \Delta_{H}$ and $S_{H}, p_{H}<p<p^{H}$}

In [14], we prove that for all $p$ in the Hodge range, the Hodge-Dirac operator has a bounded holomorphic functional calculus. We do not include a proof here, but say a little more in $\S 24$. 
Theorem 16.1. Suppose that $\Omega$ is a very weakly Lipschitz domain in $\mathbb{R}^{3}$, and that $p_{H}<p<p^{H}$, i.e. $\left(H_{p}\right) L^{p}(\Omega, \Lambda)=\mathcal{R}^{p}\left(d_{\Omega}\right) \oplus \mathcal{R}^{p}\left(\delta_{\bar{\Omega}}\right) \oplus \mathcal{N}^{p}\left(D_{H}\right)$. Then

$\left(R_{p}\right)$ The Hodge-Dirac operator $D_{H}$ is bisectorial in $L^{p}(\Omega, \Lambda)$, in particular $\left\|\left(I+i t D_{H}\right)^{-1} u\right\|_{p} \leq C\|u\|_{p} \quad \forall t \in \mathbb{R} \backslash\{0\}, \forall u \in L^{p}(\Omega, \Lambda)$;

$\left(F_{p}\right) D_{H}$ has a bounded $H^{\infty}\left(S_{\mu}^{o}\right)$ functional calculus in $L^{p}(\Omega, \Lambda)$ for all $\mu>0$, in particular, $\left\|D_{H} u\right\|_{p} \approx\left\|\sqrt{-\Delta_{H}} u\right\|_{p}$ for all $u \in \mathcal{D}^{p}\left(D_{H}\right)=\mathcal{D}^{p}\left(\sqrt{-\Delta_{H}}\right)$.

Corollary 16.2. (i) The Hodge-Laplacian $-\Delta_{H}=D_{H}{ }^{2}=d_{\Omega} \delta_{\bar{\Omega}}+\delta_{\bar{\Omega}} d_{\Omega}$ is $L^{p}$ sectorial with a bounded holomorphic functional calculus, in particular,

$\left\|\left(I-t^{2} \Delta_{H}\right)^{-1} u\right\|_{p} \leq C^{2}\|u\|_{p} \quad \forall t>0, \forall u \in L^{p}(\Omega, \Lambda)$.

(ii) The Hodge-Stokes operator $S_{H}=-\left.\Delta_{H}\right|_{\mathcal{H}^{p}}$ is sectorial with a bounded holomorphic functional calculus in $\mathcal{H}^{p}:=\left\{u \in L^{p}\left(\Omega, \Lambda^{1}\right)\right.$; $\left.\operatorname{div}_{\bar{\Omega}} u=0\right\}$, in particular,

$\left\|\left(I+t^{2} S_{H}\right)^{-1} u\right\|_{p} \leq C^{2}\|u\|_{p} \quad \forall t>0, \forall u \in \mathcal{H}^{p}$.

In the case of a bounded strongly Lipschitz domain, it was shown in [17] that $-\Delta_{H}$ and $S_{H}$ are $L^{p}$ sectorial for $p$ in an open interval containing $\left[\frac{3}{2}, 3\right]$ in dimension 3 . To our knowledge, the fact that they have a functional calculus is new, due to [14]. It was proved in [10] that for the same range of $p$ the Riesz transforms $d_{\Omega}\left(-\Delta_{H}\right)^{-\frac{1}{2}}$ and $\delta_{\bar{\Omega}}\left(-\Delta_{H}\right)^{-\frac{1}{2}}$ are bounded in $L^{p}(\Omega, \Lambda)$, again in the case of a bounded strongly Lipschitz domain.

Again: If $\Omega$ is a very weakly Lipschitz domain in $\mathbb{R}^{3}$, and $p_{H}<p<p^{H}$, then $D_{H}$, $\Delta_{H}, S_{H}$ all have $L^{p}$ resolvent bounds,

$$
\begin{array}{ll}
\left\|\left(I+i t D_{H}\right)^{-1} u\right\|_{p} \leq C\|u\|_{p} & \forall u \in L^{p}(\Omega, \Lambda), \forall t \in \mathbb{R} \backslash\{0\} \\
\left\|\left(I-t^{2} \Delta_{H}\right)^{-1} u\right\|_{p} \leq C^{2}\|u\|_{p} \quad \forall u \in L^{p}(\Omega, \Lambda), \forall t>0 \\
\left\|\left(I+t^{2} S_{H}\right)^{-1} u\right\|_{p} \leq C^{2}\|u\|_{p} \quad \forall u \in \mathcal{H}^{p}, \forall t>0
\end{array}
$$

and all have corresponding holomorphic functional calculi.

In fact, $D_{H}$ can NOT have a functional calculus in $L^{p}(\Omega, \Lambda)$ for $p$ outside the interval $\left(p_{H}, p^{H}\right)$, as shown in the Exercise in $\S 13$.

But $S_{H}$ CAN, and DOES, at least for $\max \left\{1, p_{H S}\right\}<p \leq p_{H}$ where $p_{H S}$ is the Sobolev exponent below $p_{H}$ i.e. $\frac{1}{p_{H S}}=\frac{1}{p_{H}}+\frac{1}{3}$.

Note: (i) Since $p_{H}<2$, it is easily computed that $p_{H S}<6 / 5$.

(ii) If $\Omega$ is strongly Lipschitz, then $p_{H}<3 / 2$, and so $p_{H_{S}}<1$.

\section{$17 L^{p}$ result for Hodge-Stokes operator $S_{H}$,}

$$
p_{H S}<p<p^{H}
$$

Theorem 17.1. Suppose $\Omega$ is a very weakly Lipschitz domain in $\mathbb{R}^{3}$, and $\max \left\{1, p_{H_{S}}\right\}<$ $p<p^{H}$. Then the Hodge-Stokes operator $S_{H}=-\left.\Delta_{H}\right|_{\mathcal{H}^{p}}$ is sectorial with a bounded holomorphic functional calculus in $\mathcal{H}^{p}=\left\{u \in L^{p}\left(\Omega, \Lambda^{1}\right)\right.$; $\left.\operatorname{div}_{\bar{\Omega}} u=0\right\}$. In particular,

$$
\left\|\left(I+t^{2} S_{H}\right)^{-1} u\right\|_{p} \leq C^{2}\|u\|_{p}, \quad \forall u \in \mathcal{H}^{p}, \forall t>0 .
$$


Corollary 17.2. Suppose $\Omega$ is a strongly Lipschitz domain in $\mathbb{R}^{3}$, and $1<p<p^{H}$. Then $S_{H}$ is sectorial with a bounded holomorphic functional calculus in $\mathcal{H}^{p}$.

These results are proved in [14]. Here we will not look further into functional calculi, but will indicate how to apply the fact that the Hodge-Dirac operator has $L^{q}$ resolvent bounds when $p_{H}<q<p^{H}$, to derive $L^{p}$ resolvent bounds for the Hodge-Stokes operator when $p_{H S}<p \leq p_{H}$.

The proofs depend on the theory of regularized Poincaré and Bogovskil potential operators as developed in [16] and [7] for the case when $\Omega$ is starlike or strongly Lipschitz. Here we start with the special case of the unit ball $B \subset \mathbb{R}^{3}$, and then derive what we need for very weakly Lipschitz domains.

\section{Potential operator on the unit ball}

Let

- $B=B(0,1)$, the unit ball in $\mathbb{R}^{3}$, centred at the origin;

- $\theta \in C_{c}^{\infty}\left(\frac{1}{2} B, \mathbb{R}\right)$ with $\int \theta=1$;

- $R_{B}: L^{p}(B, \Lambda) \rightarrow W^{1, p}(B, \Lambda)$, the regularized Poincaré potential operator defined by $R_{B} u=\sum_{k=1}^{3} R_{B} u^{k}$,

$$
\begin{aligned}
& \left.R_{B} u^{k}(x)=\int_{B} \theta(a)(x-a)\right\lrcorner \int_{0}^{1} t^{k-1} u^{k}(a+t(x-a)) d t d a \quad(k=1,2,3), \\
& u=\sum_{k=0}^{3} u^{k} \in L^{p}(B, \Lambda)=\oplus_{k=0}^{3} L^{p}\left(B, \Lambda^{k}\right) .
\end{aligned}
$$

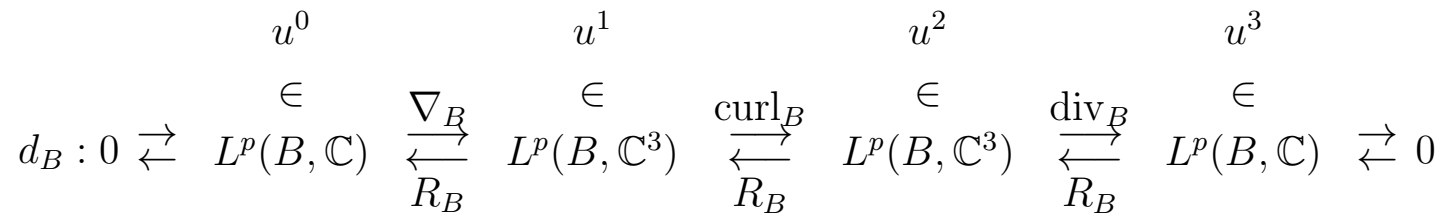

Then $R_{B}: L^{p}(B, \Lambda) \rightarrow W^{1, p}(B, \Lambda)$ is bounded, $R_{B}: L^{p}(B, \Lambda) \rightarrow L^{p}(B, \Lambda)$ is compact, and

$$
d_{B} R_{B} u+R_{B} d_{B} u+\left(\int \theta u^{0}\right) 1=u \quad \forall u \in L^{p}(B, \Lambda)
$$

(where 1 denotes the constant function $1 \in L^{p}\left(\Omega, \Lambda^{0}\right)$ ). We write this as

$$
d_{B} R_{B} u+R_{B} d_{B} u+K_{B} u=u
$$

where $K_{B} u=\left(\int \theta u^{0}\right) 1$ and note that $K_{B}: L^{p}(B, \Lambda) \rightarrow L^{\infty}\left(B, \Lambda^{0}\right)$ is bounded, and $K_{B}: L^{p}(B, \Lambda) \rightarrow L^{p}\left(B, \Lambda^{0}\right)$ is compact. The operator $K_{B}$ compensates for the fact 
that the above sequence for $d_{B}$ misses out on being exact, due to the gradient map $\nabla_{B}$ having a one dimensional null-space consisting of constant functions in $L^{p}\left(B, \Lambda^{0}\right)$.

Moreover, if $1<p=q_{S}<q<\infty$, where $p=q_{S}$ is the Sobolev exponent below $q$, i.e.

$$
\frac{1}{p}=\frac{1}{q}+\frac{1}{3}
$$

then the potential map $R_{B}: L^{p}(B, \Lambda) \rightarrow L^{q}(B, \Lambda)$ is bounded.

\section{Potential operator on bilipschitz transforma- tion of the unit ball}

Suppose $\rho: B \rightarrow \rho B \subset \mathbb{R}^{3}$ is a uniformly locally bilipschitz transformation. Then the pull-back $\rho^{*}: L^{p}(\rho B, \Lambda) \rightarrow L^{p}(B, \Lambda)$ is bounded, and

$$
d_{\rho B}=\left(\rho^{*}\right)^{-1} d_{B} \rho^{*}
$$

recall that $\left(\rho^{*} u\right)(x)=\left(\underline{\rho}_{x}\right)^{*} u(\rho(x))$ where $\underline{\rho}_{x}$ is the Jacobian matrix of $\rho$ at $x$.

Define $R_{\rho B}: L^{p}(\rho B, \Lambda) \rightarrow L^{q}(\rho B, \Lambda)$ and $K_{\rho B}: L^{p}(\rho B, \Lambda) \rightarrow L^{\infty}(\rho B, \Lambda)$ by

$$
R_{\rho B}=\left(\rho^{*}\right)^{-1} R_{B} \rho^{*} \quad \text { and } \quad K_{\rho B}=\left(\rho^{*}\right)^{-1} K_{B} \rho^{*}
$$

so that

$$
\begin{gathered}
d_{\rho B} R_{\rho B} u+R_{\rho B} d_{\rho B} u+K_{\rho B} u=u . \\
d_{\rho B}: 0 \rightleftarrows L^{p}(\rho B, \mathbb{C}) \underset{R_{\rho B}}{\stackrel{\nabla_{\rho B}}{\rightleftarrows}} L^{p}\left(\rho B, \mathbb{C}^{3}\right) \underset{R_{\rho B}}{\stackrel{\operatorname{curl}_{\rho B}}{\rightleftarrows}} L^{p}\left(\rho B, \mathbb{C}^{3}\right) \underset{R_{\rho B}}{\stackrel{\operatorname{div}_{\rho B}}{\rightleftarrows}} L^{p}(\rho B, \mathbb{C}) \rightleftarrows 0
\end{gathered}
$$

The operators $R_{\rho B}$ and $K_{\rho B}$ have the same boundedness and compactness properties as $R_{B}$ and $K_{B}$.

\section{Potential operators on very weakly Lipschitz do- mains}

- $1<p<q<\infty \quad\left(\frac{1}{p}=\frac{1}{q}+\frac{1}{3}\right)$.

- $\Omega$ is very weakly Lipschitz, i.e. $\Omega=\cup_{j=1}^{N}\left(\rho_{j} B\right)$ where each $\rho_{j}: B \rightarrow \rho_{j} B \subset \mathbb{R}^{3}$ is uniformly locally bilipschitz, and

- $1=\sum_{j=1}^{N} \chi_{j}$ on $\Omega$, where each $\chi_{j}: \Omega \rightarrow[0,1]$ is a Lipschitz function with $\operatorname{sppt}_{\Omega}\left(\chi_{j}\right) \subset \rho_{j} B$.

- Define $R_{\Omega}=\sum_{j=1}^{N} \chi_{j} R_{\rho_{j} B}$ and $K_{\Omega} u=\sum_{j=1}^{N}\left(\chi_{j} K_{\rho_{j} B} u-\left(\nabla \chi_{j}\right) \wedge R_{\rho_{j} B} u\right)$. 


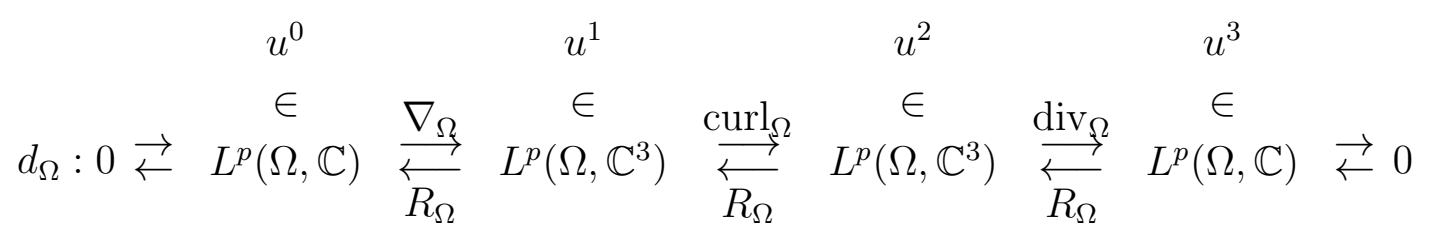

It is straightforward to apply the properties mentioned in the previous two sections to prove the following result.

Theorem 20.1. The exterior derivative $d_{\Omega}$ has a potential map $R_{\Omega}: L^{p}(\Omega, \Lambda) \rightarrow$ $L^{q}(\Omega, \Lambda)$ satisfying

$$
d_{\Omega} R_{\Omega} u+R_{\Omega} d_{\Omega} u+K_{\Omega} u=u \quad \forall u \in L^{p}(\Omega, \Lambda)
$$

where $K_{\Omega}: L^{p}(\Omega, \Lambda) \rightarrow L^{q}(\Omega, \Lambda)$. Moreover $K_{\Omega}$ and $R_{\Omega}$ are compact operators in $L^{p}(\Omega, \Lambda)$.

Remark 20.2. Although we will not use this fact in the coming sections, we remark than $R_{\Omega}$ can be modified in such a way that $d_{\Omega} R_{\Omega} u=u$ for all $u \in \mathcal{R}^{p}\left(d_{\Omega}\right)$.

Using this modification, we have that $d_{\Omega} R_{\Omega}: L^{p}(\Omega, \Lambda) \rightarrow \mathcal{R}^{p}\left(d_{\Omega}\right)$ is a bounded projection for all $p, 1<p<\infty$, and as a corollary, the spaces $\mathcal{R}^{p}\left(d_{\Omega}\right)(1<p<\infty)$ are closed subspaces of $L^{p}(\Omega, \Lambda)$ which interpolate by the complex method.

In this case, $R_{\Omega}$ is a true potential operator. For example, if $u^{1}$ is a gradient vector field, then $w_{0}=R_{\Omega} u^{1} \in L^{q}(\Omega, \mathbb{C})$ is its potential, because $\nabla_{\Omega} w_{0}=d_{\Omega} R_{\Omega} u^{1}=u^{1}$.

Remark 20.3. With a modified $R_{\Omega}$ as in Remark 20.2, define $\mathcal{Z}^{p}=K_{\Omega}\left(\mathcal{N}^{p}\left(d_{\Omega}\right)\right)$. Then $\mathcal{N}^{p}\left(d_{\Omega}\right)=\mathcal{R}^{p}\left(d_{\Omega}\right) \oplus \mathcal{Z}^{p}$ with decomposition $u=d_{\Omega} R_{\Omega} u+K_{\Omega} u$ for all $u \in$ $\mathcal{N}^{p}\left(d_{\Omega}\right)$. So the spaces in the decomposition are closed, and $\mathcal{Z}^{p}$ is finite dimensional, on account of the compactness of $K_{\Omega}$. Thus $\mathcal{R}^{p}\left(d_{\Omega}\right)$ has finite codimension in $\mathcal{N}^{p}\left(d_{\Omega}\right)$, as claimed in Section 6.

In the following section $T_{\bar{\Omega}}$ could be similarly modified to give $u=\delta_{\bar{\Omega}} T_{\bar{\Omega}} u$ for all $u \in \mathcal{R}^{p}\left(\delta_{\bar{\Omega}}\right)$.

\section{Dual potential operators}

- $1<p<q<\infty \quad\left(\frac{1}{p}=\frac{1}{q}+\frac{1}{3}\right)$;

- $T_{\bar{\Omega}}: L^{p}(\Omega, \Lambda) \rightarrow L^{q}(\Omega, \Lambda)$ is dual to $R_{\Omega}: L^{q^{\prime}}(\Omega, \Lambda) \rightarrow L^{p^{\prime}}(\Omega, \Lambda)$;

- $L_{\bar{\Omega}}: L^{p}(\Omega, \Lambda) \rightarrow L^{q}(\Omega, \Lambda)$ is dual to $K_{\Omega}: L^{q^{\prime}}(\Omega, \Lambda) \rightarrow L^{p^{\prime}}(\Omega, \Lambda)$. 
Then, dual to the equation $d_{\Omega} R_{\Omega} u+R_{\Omega} d_{\Omega} u+K_{\Omega} u=u$, is :

$$
u=\delta_{\bar{\Omega}} T_{\bar{\Omega}} u+T_{\bar{\Omega}} \delta_{\bar{\Omega}} u+L_{\bar{\Omega}} u
$$

so that $T_{\bar{\Omega}}$ is a potential operator for $\delta_{\bar{\Omega}}$, called the Bogovskiu operator :

$$
0 \leftrightarrows L^{p}(\Omega, \mathbb{C}) \underset{T_{\bar{\Omega}}}{\stackrel{-\nabla_{\bar{\Omega}}}{\leftrightarrows}} L^{p}\left(\Omega, \mathbb{C}^{3}\right) \underset{T_{\bar{\Omega}}}{\stackrel{\operatorname{curl} \bar{\Omega}}{\leftrightarrows}} L^{p}\left(\Omega, \mathbb{C}^{3}\right) \underset{T_{\bar{\Omega}}}{\stackrel{-\operatorname{div}_{\bar{\Omega}}}{\leftrightarrows}} L^{p}(\Omega, \mathbb{C}) \leftrightarrows 0: \delta_{\bar{\Omega}}
$$

\section{$22 \quad L^{p}$ results for $\Delta_{H}$ on $\mathcal{N}^{p}\left(\delta_{\bar{\Omega}}\right), p_{H_{S}}<p<p^{H}$}

Suppose that $\Omega$ is a very weakly Lipschitz domain. We have stated in Theorem 16.1 that when $p_{H}<q<p^{H}$, the Hodge-Dirac operator $D_{H}=d_{\Omega}+\delta_{\bar{\Omega}}$ is bisectorial with a bounded holomorphic funtional calculus in $L^{q}(\Omega, \Lambda)$. Our aim now is to extend this result as follows.

Theorem 22.1. Suppose that

- $p_{H}<q<p^{H}$;

- $\max \left\{1, q_{S}\right\} \leq p \leq q$ where $q_{S}$ is the lower Sobolev exponent of $q$, i.e. $\frac{1}{q_{S}}=\frac{1}{q}+\frac{1}{3}$.

Then the Hodge-Laplacian $-\Delta_{H}$ is sectorial with a bounded holomorphic functional calculus in $\mathcal{N}^{p}\left(\delta_{\bar{\Omega}}\right)=\left\{u \in L^{p}(\Omega, \Lambda) ; \delta_{\bar{\Omega}} u=0\right\}$. In particular,

$$
\left\|\left(I-t^{2} \Delta_{H}\right)^{-1} u\right\|_{p} \leq C^{2}\|u\|_{p}, \quad \forall u \in \mathcal{N}^{p}\left(\delta_{\bar{\Omega}}\right), \quad \forall t>0 .
$$

Similar resolvent bounds also holds on $\mathcal{N}\left(d_{\Omega}\right)$ and hence on $\mathcal{R}\left(\delta_{\bar{\Omega}}\right)$ and on $\mathcal{R}\left(d_{\Omega}\right)$.

On restricting to $L^{p}\left(\Omega, \Lambda^{1}\right)$, we obtain Theorem 17.1 as a corollary.

For the results on functional calculi, we refer the reader to [14]. We do not fully prove the resolvent bounds either, but give the spirit of the method by outlining the estimates in the case when $p=q_{S}$.

\section{$23 L^{p}$ resolvent bounds for $\Delta_{H}$ on $\mathcal{N}^{p}\left(\delta_{\bar{\Omega}}\right), p=q_{S}$, $p_{H}<q<p^{H}$}

- $\Omega$ is very weakly Lipschitz and $p_{H}<q<p^{H}, p=q_{S}>1$.

- The idea is to modify the techniques of Blunck-Kunstmann [6], but there is still quite a bit to do, because we are working on the subspace $\mathcal{N}^{p}\left(\delta_{\bar{\Omega}}\right)$. We will not consider the functional calculus here, but will outline a proof of resolvent bounds. 
- The easy part: When $t \geq 1$, and $\delta_{\bar{\Omega}} u=0$, then

$$
\begin{aligned}
\left\|\left(I-t^{2} \Delta_{H}\right)^{-1} u\right\|_{p} & \lesssim\left\|\left(I-t^{2} \Delta_{H}\right)^{-1} u\right\|_{q} \quad \text { (because } \Omega \text { is bounded) } \\
& =\left\|\left(I-t^{2} \Delta_{H}\right)^{-1}\left(\delta_{\bar{\Omega}} T_{\bar{\Omega}}+L_{\bar{\Omega}}\right) u\right\|_{q} \\
& \leq t\left\|\delta_{\bar{\Omega}}\left(I-t^{2} \Delta_{H}\right)^{-1} T_{\bar{\Omega}} u\right\|_{q}+\left\|\left(I-t^{2} \Delta_{H}\right)^{-1} L_{\bar{\Omega}} u\right\|_{q} \\
& \lesssim\left\|t D_{H}\left(I+t^{2} D_{H}{ }^{2}\right)^{-1} T_{\bar{\Omega}} u\right\|_{q}+\left\|\left(I+t^{2} D_{H}{ }^{2}\right)^{-1} L_{\bar{\Omega}} u\right\|_{q} \\
& \lesssim\left\|T_{\bar{\Omega}} u\right\|_{q}+\left\|L_{\bar{\Omega}} u\right\|_{q} \\
& \lesssim\|u\|_{p}
\end{aligned}
$$

(using Hodge decomposition in $L^{q}(\Omega, \Lambda)$ in line 4, and resolvent bounds for $D_{H}$ in $L^{q}(\Omega, \Lambda)$ in line 5$)$.

- Henceforth take $0<t<1$.

- Cover $\Omega$ : Let $\underline{Q}_{j}^{t}(j \in J)$ be the cubes in $\mathbb{R}^{3}$ with side-length $t$ and corners at points in $t \mathbb{Z}^{3}$, which intersect $\Omega$. Let $Q_{j}^{t}=4 \underline{Q}_{j}^{t} \cap \Omega$. Then $\Omega=\cup Q_{j}^{t}$. Write $1=\sum_{j \in J} \eta_{j}^{2}$ on $\Omega$, where $\eta_{j} \in C_{c}^{1}\left(4 \underline{Q}_{j}^{t},[0,1]\right)$ and $\left\|\nabla \eta_{j}\right\|_{\infty} \leq 1 / t$. The "cubes" $Q_{j}^{t}$ have finite overlap, in fact $\sum_{j \in J} 1_{Q_{j}^{t}} \leq 64$. (Here $1_{Q_{j}^{t}}$ denotes the function with value 1 on $Q_{j}^{t}$ and zero elsewhere on $Q$.)

- $L^{q}$ off-diagonal bounds in $\operatorname{dist}\left(Q_{j}^{t}, Q_{k}^{t}\right)=\inf \left\{|x-y| ; x \in Q_{j}^{t}, y \in Q_{k}^{t}\right\}$ are a consequence of the $L^{q}$ resolvent bounds. See [14, §5], or adapt the $L^{2}$ proofs in [4]. We need the following two bounds.

For each $N \in \mathbb{N}$, there exists $C_{N}$ such that, when $\operatorname{sppt}(f) \in Q_{k}^{t}$, then

$$
\begin{aligned}
\left\|1_{Q_{j}^{t}}\left(I-t^{2} \Delta_{H}\right)^{-1} f\right\|_{q} & \leq C_{N}\left(\frac{t}{t+\operatorname{dist}\left(Q_{j}^{t}, Q_{k}^{t}\right)}\right)^{N}\|f\|_{q} \quad \text { and } \\
t\left\|1_{Q_{j}^{t}}\left(I-t^{2} \Delta_{H}\right)^{-1} \delta_{\bar{\Omega}} f\right\|_{q} & \leq C_{N}\left(\frac{t}{t+\operatorname{dist}\left(Q_{j}^{t}, Q_{k}^{t}\right)}\right)^{N}\|f\|_{q} .
\end{aligned}
$$

- Decompose $u \in \mathcal{N}^{p}\left(\delta_{\bar{\Omega}}\right)$ (using $\left.\left.\delta_{\bar{\Omega}}\left(\eta_{k} f\right)-\eta_{k} \delta_{\bar{\Omega}} f=\left(\nabla \eta_{k}\right)\right\lrcorner f\right)$ :

$$
\begin{aligned}
u & =\sum_{k \in J} \eta_{k}^{2} u=\sum_{k \in J} \eta_{k} I \eta_{k} u \\
& =\sum_{k \in J}\left(\eta_{k} \delta_{\bar{\Omega}} T_{\bar{\Omega}} \eta_{k} u+\eta_{k} T_{\bar{\Omega}} \delta_{\bar{\Omega}} \eta_{k} u+\eta_{k} L_{\bar{\Omega}} \eta_{k} u\right) \\
& \left.\left.=\sum_{k \in J}\left(\delta_{\bar{\Omega}}\left(\eta_{k} T_{\bar{\Omega}} \eta_{k} u\right)-\left(\nabla \eta_{k}\right)\right\lrcorner T_{\bar{\Omega}} \eta_{k} u+\eta_{k} T_{\bar{\Omega}}\left(\nabla \eta_{k}\right)\right\lrcorner u+\eta_{k} L_{\bar{\Omega}} \eta_{k} u\right) \\
& =\sum_{k \in J}\left(\delta_{\bar{\Omega}} w_{k}+\frac{1}{t} v_{k}\right) \quad \text { where } \\
w_{k} & =\eta_{k} T_{\bar{\Omega}} \eta_{k} u \quad \text { and } \\
v_{k} & \left.\left.=-\left(t \nabla \eta_{k}\right)\right\lrcorner T_{\bar{\Omega}} \eta_{k} u+\eta_{k} T_{\bar{\Omega}}\left(t \nabla \eta_{k}\right)\right\lrcorner u+t \eta_{k} L_{\bar{\Omega}} \eta_{k} u .
\end{aligned}
$$


- On using the $L^{p}-L^{q}$ bounds on $T_{\bar{\Omega}}$ and $L_{\bar{\Omega}}$, we obtain

$$
\begin{aligned}
&\left\|w_{k}\right\|_{q} \lesssim\left\|\eta_{k} u\right\|_{p} \lesssim\left\|1_{Q_{k}^{t}} u\right\|_{p} \quad \text { with } \operatorname{sppt}\left(w_{k}\right) \subset Q_{k}^{t} \quad \text { and } \\
&\left\|v_{k}\right\|_{q} \lesssim(1+t)\left\|1_{Q_{k}^{t}} u\right\|_{p} \lesssim\left\|1_{Q_{k}^{t}} u\right\|_{p} \quad \text { with } \operatorname{sppt}\left(v_{k}\right) \subset Q_{k}^{t} .
\end{aligned}
$$

Here now is the resolvent estimate. Suppose $\delta_{\bar{\Omega}} u=0$. Then

$$
\begin{aligned}
& \left\|\left(I-t^{2} \Delta_{H}\right)^{-1} u\right\|_{p} \leq\left[\sum_{j \in J} \int_{Q_{j}^{t}}\left|\left(I-t^{2} \Delta_{H}\right)^{-1} u\right|^{p}\right]^{\frac{1}{p}} \\
& =\left[\sum_{j \in J}\left(\left\|1_{Q_{j}^{t}}\left(I-t^{2} \Delta_{H}\right)^{-1} u\right\|_{p}\right)^{p}\right]^{\frac{1}{p}} \\
& \leq\left[\sum_{j \in J}\left(\left\|1_{Q_{j}^{t}}\left(I-t^{2} \Delta_{H}\right)^{-1} u\right\|_{q}\left|Q_{j}^{t}\right|^{\frac{1}{3}}\right)^{p}\right]^{\frac{1}{p}} \quad\left(\frac{1}{p}=\frac{1}{q}+\frac{1}{3}\right) \\
& \lesssim\left[\sum_{j \in J}\left(\sum_{k \in J}\left\|1_{Q_{j}^{t}}\left(I-t^{2} \Delta_{H}\right)^{-1}\left(\delta_{\bar{\Omega}} w_{k}+\frac{1}{t} v_{k}\right)\right\|_{q} t\right)^{p}\right]^{\frac{1}{p}} \\
& \lesssim\left[\sum_{j \in J}\left(\sum_{k \in J}\left(\frac{t}{t+\operatorname{dist}\left(Q_{j}^{t}, Q_{k}^{t}\right)}\right)^{4}\left(\left\|w_{k}\right\|_{q}+\left\|v_{k}\right\|_{q}\right)\right)^{p}\right]^{\frac{1}{p}} \\
& \lesssim\left[\sum_{j \in J}\left(\sum_{k \in J}\left(\frac{t}{t+\operatorname{dist}\left(Q_{j}^{t}, Q_{k}^{t}\right)}\right)^{4}\left\|1_{Q_{k}^{t}} u\right\|_{p}\right)^{p}\right]^{\frac{1}{p}} \\
& \lesssim\left(\sup _{j} \sum_{k \in J}\left(\frac{t}{t+\operatorname{dist}\left(Q_{j}^{t}, Q_{k}^{t}\right)}\right)^{4}\right)\left[\sum_{k \in J}\left\|1_{Q_{k}^{t}} u\right\|_{p}^{p}\right]^{\frac{1}{p}} \\
& \lesssim\left[\sum_{k \in J}\left\|1_{Q_{k}^{t}} u\right\|_{p}^{p}\right]^{\frac{1}{p}}=\left[\sum_{k \in J} \int_{Q_{k}^{t}}|u|^{p}\right]^{\frac{1}{p}} \\
& =\left(\int_{\Omega} \sum_{k \in J} 1_{Q_{k}^{t}}|u|^{p}\right)^{\frac{1}{p}} \lesssim\|u\|_{p}
\end{aligned}
$$

as claimed.

- In $\left(^{*}\right)$ we used the off-diagonal bounds with $N=4$;

- In $(* *)$ we used the Schur estimate in $\ell^{p}(J)$, with $A_{j, k}=\left(\frac{t}{t+\operatorname{dist}\left(Q_{j}^{t}, Q_{k}^{t}\right)}\right)^{4}$ and $\beta_{k}=\left\|1_{Q_{k}^{t}} u\right\|_{p}$ :

$$
\begin{aligned}
{\left[\sum_{j}\left|\sum_{k} A_{j, k} \beta_{k}\right|^{p}\right]^{\frac{1}{p}} } & \leq\left(\sup _{j} \sum_{k}\left|A_{j, k}\right|\right)^{\frac{1}{p^{\prime}}}\left(\sup _{k} \sum_{j}\left|A_{j, k}\right|\right)^{\frac{1}{p}}\left(\sum_{k}\left|\beta_{k}\right|^{p}\right)^{\frac{1}{p}} \\
& =\left(\sup _{j} \sum_{k}\left|A_{j, k}\right|\right)\left(\sum_{k}\left|\beta_{k}\right|^{p}\right)^{\frac{1}{p}} \quad \text { when } A_{j, k}=A_{k, j}
\end{aligned}
$$


- In (***) we used that, given $Q_{j}^{t}$,

$$
\begin{aligned}
\sum_{k}\left(\frac{t}{t+\operatorname{dist}\left(Q_{j}^{t}, Q_{k}^{t}\right)}\right)^{4} & \lesssim C_{0}+\sum_{M=0}^{\infty} \sum_{\left\{k ; 2^{M} t \leq \operatorname{dist}\left(Q_{j}^{t}, Q_{k}^{t}\right)<2^{(M+1)} t\right\}} \frac{1}{2^{4 M}} \\
& \lesssim C_{0}+\sum_{M=0}^{\infty} 2^{3 M} \frac{1}{2^{4 M}}=C_{0}+\sum_{M=0}^{\infty} \frac{1}{2^{M}} \leq C
\end{aligned}
$$

- In $(* * * *)$ we used the finite overlap of the cubes.

This completes the proof of (1) in the case when $p=q_{S}$. The proof of $L^{p}$ sectoriality when $q_{S} \leq p<q$ requires minor modification. To show that $S_{H}$ has a bounded holomorphic functional calculus requires further work, using a CalderónZygmund decomposition of $\Omega$. For this, the reader is referred to [14].

\section{Remarks on obtaining resolvent bounds in the Hodge range}

In the previous section we applied Theorem 16.1. But suppose we just start with the $L^{2}$ resolvent bounds. Then a similar procedure to that described above, can be used to obtain resolvent bounds for $D_{H}$ on $\mathcal{N}^{p}\left(\delta_{\bar{\Omega}}\right)$ when $6 / 5=2_{S} \leq p \leq 2$ Moreover, use of the potential operators $R_{\Omega}$ will lead to resolvent bounds on $\mathcal{N}^{p}\left(d_{\Omega}\right)$, also when $6 / 5 \leq p \leq 2$. Now, if $p$ is also in the Hodge range, we then obtain resolvent bounds on all of $L^{p}(\Omega, \Lambda)$, i.e. we obtain resolvent bounds for $D_{H}$ on $L^{p}(\Omega, \Lambda)$ when $\max \left\{6 / 5, p_{H}\right\}<p \leq 2$. Repeating this procedure once more if necessary, we obtain resolvent bounds on $L^{p}(\Omega, \Lambda)$ for $p_{H}<p \leq 2$ (as $\left.(6 / 5)_{S}<1\right)$. A duality argument then gives resolvent bounds when $2 \leq p<p^{H}$. In this way, the statement $\left(R_{p}\right)$ can be proved when $p_{H}<p<p^{H}$, as stated in Theorem 16.1. See [14] for details.

We remark that such an iteration method has been used previously in [9] in the study of more general first order systems on $\mathbb{R}^{n}$. A similar iteration procedure has been used also in [17] and [10].

\section{Parabolic equations}

As mentioned in $\S 14$, operators with a bounded holomorphic functional calculus on a closed subspace $\mathcal{X}^{p}$ of $L^{p}(\Omega, \Lambda)$, also satisfy maximal regularity. So, on taking $\mathcal{X}^{p}=\mathcal{H}^{p}$, we obtain:

Theorem 25.1. Suppose that $\Omega$ is a very weakly Lipschitz domain in $\mathbb{R}^{3}$, that $\max \left\{1, p_{H_{S}}\right\}<p<p^{H}$, and that $1<q<\infty, 0<T \leq \infty$. Suppose also that

$$
\begin{aligned}
\partial_{t} F(t, .)+S_{H} F(t, .) & =h(t, .) \in L^{q}\left((0, T) ; \mathcal{H}^{p}\right), t>0 \\
F(0, .) & =0 .
\end{aligned}
$$


Then

$$
\left\{\int_{0}^{T}\|F(t, .)\|_{p}^{q} d t\right\}^{1 / q}+\left\{\int_{0}^{T}\|S F(t, .)\|_{p}^{q} d t\right\}^{1 / q} \leq C_{p, q}\left\{\int_{0}^{T}\|h(t, .)\|_{p}^{q} d t\right\}^{1 / q} .
$$

\section{References}

[1] D. Albrecht, X. Duong, A. McIntosh. Operator theory and harmonic analysis. In: Instructional Workshop on Analysis and Geometry, Part III (Canberra, 1995), Proc. Centre Math. Appl. Austral. Nat. Univ. 34 (1996) 77-136. 14

[2] P. Auscher, A. McIntosh, A. Nahmod. Holomorphic functional calculi of operators, quadratic estimates and interpolation. Indiana Univ. Math. J. 46 (1997) 375-403. 14

[3] P. Auscher, A. McIntosh, E. Russ. Hardy Spaces of Differential Forms on Riemannian Manifolds. J. Geom. Anal. 18 (2008) 192-248. 14

[4] A. Axelsson, S. Keith, A. McIntosh. Quadratic estimates and functional calculi of perturbed Dirac operators. Invent. math. 163 (2006) 455-497. 23

[5] Andreas Axelsson and Alan McIntosh. Hodge decompositions on weakly Lipschitz domains. Advances in Analysis and Geometry, 3-29, Trends Math., Birkhäuser, Basel (2004) 3-29. 4

[6] S. Blunck and P. Kunstmann. Calderón-Zygmund theory for non-integral operators and the $H^{\infty}$ functional calculus. Rev. Mat. Iberoamericana, 19 (2003), 919942. 23

[7] Martin Costabel and Alan McIntosh. On Bogovskiu and regularised Poincaré operators for de Rham complexes on Lipschitz domains. Math Z. 265 (2010) 297320. 17

[8] M. Cowling, I. Doust, A. McIntosh, A. Yagi. Banach space operators with a bounded $H^{\infty}$ functional calculus. J. Austral. Math. Soc. Ser. A 60 (1996) 51-89. 14

[9] D. Frey, A. McIntosh and P. Portal. Conical square function estimates and functional calculi for perturbed Hodge-Dirac operators in $L^{p}$. Journal d'Analyse Mathématique, to appear; http://arxiv.org/abs/1407.4774 24

[10] S. Hofmann, M. Mitrea, and S. Monniaux. Riesz transforms associated with the Hodge Laplacian in Lipschitz subdomains of Riemannian manifolds. Ann. Inst. Fourier (Grenoble), 61(4) (2012) 1323-1349. 16, 24

[11] Tuomas Hytönen, Alan McIntosh. Stability in $p$ of the $H^{\infty}$-calculus of first-order systems in $L^{p}$. Proc. Centre Math. Appl. Austral. Nat. Univ. 44 (2010) 167-181. 15 
[12] P. C. Kunstmann, L. Weis. Maximal $L_{p}$ regularity for parabolic problems, Fourier multiplier theorems and $H^{\infty}$-functional calculus, Lect. Notes in Math. 1855. Springer-Verlag (2004). 14

[13] Alan McIntosh. Operators which have an $H^{\infty}$ functional calculus. Proc. Centre Math. Appl. Austral. Nat. Univ. 14 (1986) 210-231. 14

[14] Alan McIntosh and Sylvie Monniaux. Hodge-Dirac, Hodge-Laplacian and HodgeStokes operators in $L^{p}$ spaces on Lipschitz domains. In preparation 1, 15, 16, 16, $17,22,23,23,24$

[15] Marius Mitrea. Sharp Hodge decompositions, Maxwell's equations, and vector Poisson problems on nonsmooth, three-dimensional Riemannian manifolds. Duke Math. J. 125(3) (2004) 467-547. 15

[16] D. Mitrea, M. Mitrea and S. Monniaux. The Poisson problem for the exterior derivative operator with Dirichlet boundary condition on nonsmooth domains. Commun. Pure Appl. Anal. 7 (2008) 1295-1333. 7.1, 17

[17] M. Mitrea and S. Monniaux. On the analyticity of the semigroup generated by the Stokes operator with Neumann-type boundary conditions on Lipschitz subdomains of Riemannian manifolds. Trans. Amer. Math. Soc. 361(6) (2009) 3125-3157. 11, 16,24

[18] Günter Schwarz. Hodge decomposition-a method for solving boundary value problems. Lecture Notes in Mathematics, vol. 1607, Springer-Verlag, Berlin, 1995. 15 